DOI:

10.1038/nrn2227

\title{
The endocytic puzzle
}

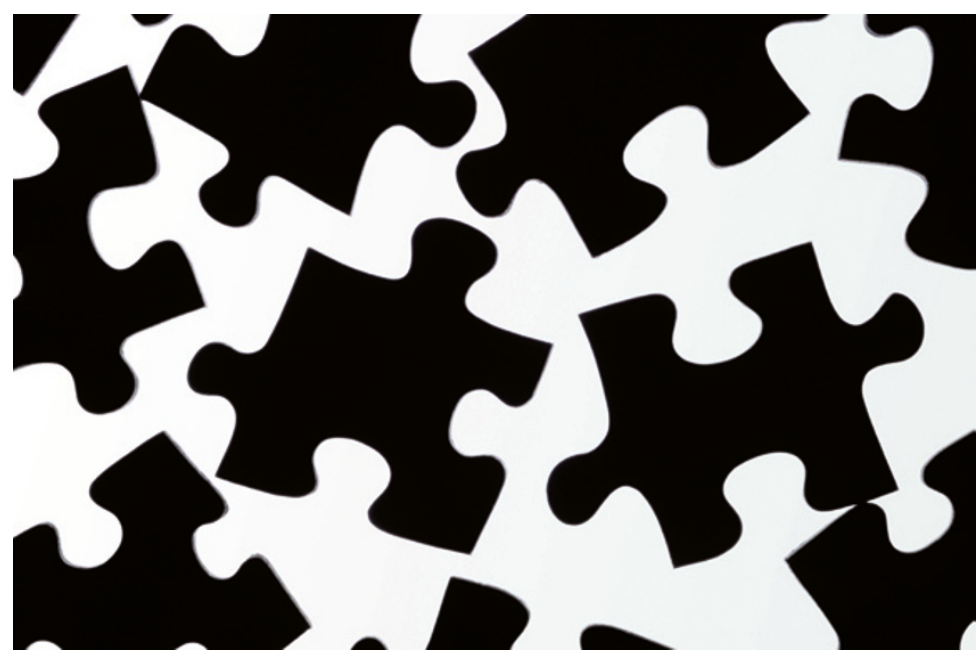

Endocytosis is dependent on the functional interaction of numerous proteins that assist in the efficient retrieval of selected membrane components. Although several components of the endocytic machinery are well characterized, others remain to be functionally analysed. In their recent paper in the Journal of Cell Biology, Koh et al. showed that epidermal growth factor receptor pathway substrate clone 15 (Eps15) controls synaptic vesicle endocytosis and is also involved in synapse development in Drosophila.

The authors used Drosophila eps 15 mutants to elucidate the role of Eps15 in endocytosis and development. The eps15 null-mutation itself was lethal. However, the phenotype could be rescued by introducing eps15 under the control of a neuronal -specific driver, suggesting that the vital role of Eps 15 is restricted to the nervous system.

Eps15 immunoprecipitates with a number of endocytic proteins. The authors studied the subcellular localization of Eps15 at neuromuscular junctions (NMJs), as these synapses are easily accessible in both larvae and adult flies. They found that, at resting synapses, Eps15 was associated with vesicles in the presynaptic terminal but was virtually excluded from exocytic sites. Upon stimulation, enhanced Eps15 labelling was detected in regions that are thought to be endocytic zones adjacent to these sites. In the Drosophila shibire mutant, which is characterized by large invaginations at endocytic sites, Eps 15 was associated with these invaginations. These findings implicate Eps15 in the endocytic process.

Electrophysiological experiments showed that in eps 15 mutants synaptic transmission is severely impaired, but only during intense stimulation. This indicates that Eps15 is essential for efficient membrane retrieval under stringent conditions. Similar electrophysiological phenotypes were found in eps 15 mutants, dap 160 mutants and eps15 dap160 double mutants, suggesting that both proteins contribute to the same molecular process.

The authors also found that the levels of other proteins that contribute to the endocytic machinery, such as Dap160 and dynamin, were dramatically downregulated at the NMJs of eps 15 mutants, whereas Stoned B, $\alpha$-adaptin, synaptotagmin I and endophilin were less, but nevertheless still significantly, downregulated. These findings point to a role for Eps15 in maintaining proteins at the synaptic site. A surprising finding was that although Eps15 has a conserved direct interaction with $\alpha$-adaptin, this interaction is not required for the role of Eps15 in synaptic vesicle endocytosis.

The authors also investigated the function of Eps15 during the development of NMJs in eps 15 mutants. During Drosophila development, the NMJ is a dynamic structure that consists of numerous interconnected boutons, which are sometimes branched. In eps15-null mutants, developing NMJs showed more branchpoints and a higher number of boutons than in wild-type flies.

The authors propose that Eps15 has a dual role in endocytosis and synapse development. Further studies will be needed to elucidate the signalling pathways of these two functions.

Claudia Wiedemann

ORIGINAL RESEARCH PAPER Koh, T.-W., Korolchuk, V. I. et al. Eps15 and Dap160 control synaptic vesicle membrane retrieval and synapse development. J. Cell Biol. 178, 309-322 (2007) 